Arteterapia. Papeles de arteterapia y educación para inclusión social ISSN-e 1988-8309

http://dx.doi.org/10.5209/ARTE.60119

\title{
El poder terapéutico de la dramatización: estimulación de las neuronas espejos implicadas en el lenguaje a través de emoción
}

\author{
Laura Martín Martínez
}

Recibido: 04 de mayo de 2018 / Aceptado: 24 de mayo de 2018

Resumen. Actualmente nos encontramos con la inclusión de algunos menores en los programas de Necesidades Educativas Especiales (NEE) debido a las dificultades que presentan en el desarrollo y adquisición del lenguaje. Los programas de NEE como las terapias tradicionales empleadas en trastornos del lenguaje ponen de manifiesto las limitaciones que presentan. Existen terapias alternativas, basadas en el arteterapia, no invasivas, capaces de mejorar afecciones del lenguaje estimulando los sentidos, la creatividad o las emociones.

En este artículo nos centramos en un menor con problemas en la adquisición del lenguaje y varias dificultades de diferente índole. Para esta intervención se diseña y aplican sesiones de dramatización y en concreto de Teatro de Sombras con la finalidad de actuar y estimular el área cognitiva, motora, el área de lenguaje y la comunicación y el área socioemocional, y de esta forma intervenir de una manera global en el menor. Destacamos como la estimulación de las neuronas espejo tiene una enorme incidencia en este tipo de intervenciones.

Con el propósito de conocer si, tras la intervención de Teatro de Sombras y la estimulación de las denominadas neuronas espejo, existen mejoras en la intención comunicativa, en el área propioceptiva, lingüística y emocional del menor, se diseña y aplica un estudio experimental prospectivo de caso único. Se ha aplicado un estudio de caso como método de investigación, mediante la recopilación de datos que nos permitan obtener resultados del estudio. Este estudio se considera evaluativo pretendiendo evaluar, mediante la recogida y análisis de los datos, la intervención de Teatro de Sombras para emitir juicios y conclusiones sobre la misma. La recopilación y el análisis de los datos registrados en dicho estudio, nos permite concluir, que existe la posibilidad de obtener, mediante el Teatro de Sombras mejoras en la intención comunicativa del menor, animándonos a seguir investigando en esta línea de intervenciones alternativas.

Palabras clave: arteterapia; drama; neuronas espejo; lenguaje.

\section{[en] The therapeutic power of dramatization: stimulation of neurons mirrors involved in language through emotion}

Abstract. Currently we find the inclusion of some children in the programs of Special Educational Needs (SEN) due to the difficulties they have in the development and acquisition of language. SEN programs such as traditional therapies used in language disorders highlight the limitations they present. There are alternative therapies, based on art therapy, non-invasive, capable of improving language disorders by stimulating senses, creativity or emotions.

In this article we focus on the child of a child with problems in the acquisition of language and various difficulties of different kinds. For this intervention dramatization sessions are designed and applied, specifically the Shadow Theater, with the purpose of acting and stimulating the cognitive, motor, language and communication area and the socio-emotional area, and thus intervene in a way global in the minor. We emphasize how the stimulation of mirror neurons has an enormous incidence in this type of interventions.

With the purpose of knowing if, after the intervention of Shadow Theater and the stimulation of the so-called mirror neurons, there are improvements in the communicative intention, in the minor's 
proprioceptive, linguistic and emotional area, a prospective experimental study of the child is designed and applied.

A case study has been applied as a research method, through the collection of data that allow us to obtain results of the study. This study is considered evaluative trying to evaluate, through the collection and analysis of data, the intervention of Shadow Theater to issue judgments and conclusions about it. The compilation and analysis of the data recorded in this study, allows us to conclude that there is the possibility of obtaining, through the Shadow Theater, improvements in the communicative intention of the minor, encouraging us to continue investigating in this line of alternative interventions.

We hope to give more light on mirror neurons, and the possibility that it can offer us in affected children in the acquisition of language.

Keywords: Art therapy; drama; mirror neurons; language.

Sumario: 1. Introducción. 2. El poder del arte en las neuronas espejo. 3. Objetivo del estudio. 4. Método del estudio. 5. Muestra seleccionada. 6. Instrumentos utilizados. 7. Procedimiento. 8. Resultados obtenidos. 9. Valoraciones externas 10. Conclusión. 11. Referencias bibliográficas.

Cómo citar: Martín Martínez, L. (2018). El poder terapéutico de la dramatización: estimulación de las neuronas espejos implicadas en el lenguaje a través de emoción. El poder terapéutico de la dramatización: estimulación de las neuronas espejos implicadas en el lenguaje a través de emoción, en Arteterapia. Papeles de arteterapia y educación para inclusión social 13, 2018, 33-52.

\section{Introducción}

Este artículo es un extracto de una tesis doctoral que investiga la mejora del alumnado con dificultades en la adquisición del lenguaje mediante el uso de medios innovadores, a través de una intervención procedente de las Artes. La tesis doctoral estudia nueve niños afectados por diversas patologías, con el nexo común de estar afectados en la adquisición y/o el desarrollo del lenguaje. Extraemos uno de ellos para exponerlo en este artículo, con la finalidad de avanzar en el estudio de terapias alternativas en la estimulación del lenguaje. Cabe destacar que el niño, además de tener afectada la adquisición y/o el desarrollo del lenguaje, presenta dificultades en el control postural, en el lenguaje espontáneo, baja autoestima, escasa conciencia corporal y dificultades en las relaciones sociales.

Esta investigación trata de implicar al cuerpo en su globalidad en la educación de los niños. El niño ha de sentir la vinculación entre su cuerpo y sus emociones alcanzando así un sano desarrollo psíquico, físico y emocional. En la escuela, a pesar de ser consciente de la existencia de su cuerpo, el niño contiene sus impulsos, reprimiendo su cuerpo y adiestrando sus emociones.

En el mundo actual el juego creativo tiende a desaparecer, siendo los niños guiados en el juego por personas especializadas. Los niños van perdiendo la capacidad para jugar, imaginar, inventar, de dedicarse al juego en cuerpo y alma, de evadirse del mundo real viajando a su mundo real imaginado (Chavarría, 2015).

En el juego dramático la improvisación del niño juega un papel fundamental. Intervienen el lenguaje verbal, la expresión artística, el ritmo, el cuerpo y de esta forma el niño es capaz de expresar emociones, liberar tensiones, resolver conflictos y relacionarse con los otros niños participantes (Cruz, Caballero, y Ruiz, 2013).

Moreno (1995) explica como mediante la dramatización el individuo es capaz de reconstruir su imagen para recomponer una nueva percepción de sí mismo. La 
dramatización le permite al individuo reinventarse, la sombra proyecta nuevas percepciones, estos impactos visuales generan desconexiones y nuevas conexiones cerebrales que conducen a una nueva toma de conciencia. El cerebro tiene que moldear nuevos pensamientos, pero para que perduren en el tiempo hay que vivenciarlos en el propio cuerpo, esto es posible gracias al poder del arte que emerge de la dramatización.

La actuación dramática es un estado interior intermedio, un intermediario entre la fantasía y la realidad. La modalidad es ficticia pero la experiencia es muy real. La modalidad ficticia nos permite hacer cosas que aún se encuentran afuera de nuestro alcance en la vida real, tales como expresar emociones temidas, cambiar patrones de conducta o exhibir nuevos rasgos. Una vez que las hemos vivenciado, aunque en modo ficticio, estas nuevas experiencias pueden formar parte del repertorio de nuestra vida real. (Emunah, 1994, p. 27)

El Teatro de Sombras puede actuar como lugar terapéutico que retroalimenta nuestro estado emocional, expulsa a los fantasmas y los descubre para establecer nuevos vínculos mediante la interpretación de roles en conflicto. Reinterpretarnos mediante nuestra sombra nos permite transformarnos mediante la acción dramática para reelaborar, restructurar y reintegrar esta nueva concepción de nosotros mismos.

Para Martín y López (2007):

El teatro de sombras es un recurso extraordinariamente interesante para trabajar la capacidad expresiva de nuestro alumnado, además de otros contenidos propios de la psicomotricidad y el currículum oficial de educación infantil (p.45)

Este estudio comienza desde el deseo de diseñar y aplicar una intervención de Teatro de Sombras como una posible alternativa terapéutica mediante la cual, menores con trastornos del lenguaje, sean capaces de liberar, a través del cuerpo, sus sentimientos y emociones mediante estímulos tónico-emocionales sin restricciones.

\section{El poder del arte en las neuronas espejo}

Peter Brook (2002), prestigioso director de teatro, nos habla de la vinculación del cuerpo con el mundo exterior, de cómo los actores nos muestran acciones reales, situaciones que pueden ocurrir en cualquier momento en la vida real y llevando estas situaciones a escena, conmover al espectador. Para Brook (2002) el teatro nos hace presente lo ausente. Nos habla del teatro como un espacio vacío, dónde nada parece ser material, pero que está repleto de energías invisibles. Esas energías se hacen perceptibles a través de la actuación del actor. Hace visibles esas energías. Hace partícipe al espectador de la ficción ofrecida dentro de la obra.

Para Brook (2002) el teatro es como una puerta que se abre dando la posibilidad de que estén en la misma sintonía todos los intervinientes, conectando a actores, 
creadores, espectadores, y de esta forma estimular la imaginación, las emociones y el intelecto de cada uno de ellos, consiguiendo que todos sean a la vez creadores y observadores. Nos hace conscientes de lo inconsciente, viviendo esos momentos mágicos que el teatro nos ofrece.

El arte en general y el teatro en concreto, tiene el poder de estimular las emociones, favoreciendo la adquisición del lenguaje. El niño consigue configurar su lenguaje a través de la conciencia corporal, la imagen y la percepción de sí mismo.

A través del arte, el niño logra adquirir la concepción de sí mismo de forma plena. A través del teatro el niño se sumerge en su experiencia artística, concibe su propio cuerpo en su absoluta totalidad, no discierne su dificultad de expresión verbal como estamos acostumbrados a ver en terapias tradicionales.

El arte posee un poder terapéutico capaz de despertar la emoción. Damasio definió la emoción de la siguiente manera:

Veo la esencia de la emoción como el conjunto de cambios en el estado corporal que son inducidos en multitud de órganos por los terminales de las neuronas, bajo el control de un sistema cerebral dedicado, que está respondiendo al contenido de pensamientos en relación a una entidad o acontecimientos determinados. (Damasio, 2011, p. 199)

Mediante la dramatización, el niño vivencia su propia imagen corporal, y así, de forma inconsciente activa indirectamente diferentes áreas sensoriales implicadas en el desarrollo del lenguaje, es decir se genera un poder curativo. El arte es el hilo conductor entre la realidad y la emoción.

Para una correcta asimilación del lenguaje, es preciso el estímulo proveniente de su entorno. El arte tiene ese poder de estimulación, lográndolo simultáneamente en multitud de órganos y terminales neuronales que estimulan, a su vez, al propio lenguaje.

Desde la experiencia artística, el niño adquiere la "sensación de sí mismo" que definía Damasio (2011) como las huellas fisiológicas que la experiencia deposita en el cuerpo. La experiencia artística despierta la emoción y estimula la sinapsis (proceso de comunicación entre neuronas mediante procesos eléctricos y elementos bioquímicos). Las neuronas responden a los estímulos que percibimos, por lo tanto el arte, y su extraordinario poder de estimulación, es capaz de provocar dichos estímulos de forma significativa "conformando circuitos neuronales especializados funcionalmente que generan patrones de actividad que se nutren de la información sensorio motriz que representamos mentalmente." (Luna, 2008, p. 16).

Podemos afirmar que el arte activa mediante estímulos el sistema sensorio motriz y dichos estímulos son retenidos por las neuronas sensoriales para transformarlos en señales eléctricas y químicas (este proceso se conoce como transducción). Las neuronas responden de diferente manera a los estímulos naturales y a los estímulos emocionales significativos. Estos últimos son desencadenados por la expresión artística y son detectados antes incluso que la atención selectiva. La amígdala, que se encuentra junto al hipotálamo, abarca las estructuras del sistema límbico y de los circuitos que generan la emoción, la motivación y del control del sistema nerviosos autónomo. Con las descargas de emociones, se producen respuestas químicas recibidas por distintas estructuras, modificándose así las funciones de las vísceras, los órganos 
y el sistema nervioso central. Estos circuitos neuronales implicados en la emoción, organizan y modifican el control de la "función cardiovascular y respiratoria siendo responsable de la función del control de los movimientos de la laringe, la lengua, la cara y faringe en el que la plasticidad cerebral permite asumir nuevas funciones para perfeccionar sus partes." (Luna, 2008, p. 24)

No podemos, por tanto, desvincular la emoción de los procesos cognitivos, motores y sensoriales implicados en el lenguaje. Este es ordenado y organizado mediante imágenes para explicar y comprender la realidad. Para que el niño asimile de forma correcta el lenguaje, lo desarrolle de forma natural y consiga su correcta adquisición, se precisa del estímulo que proviene de su entorno.

Debemos despertar el interés del niño y así posibilitar el aprendizaje del lenguaje. Que mejor manera de captar su atención que mediante canales implícitos que se basan en despertar la emoción y en el movimiento. Se trata de, mediante los sentidos, recibir la información y esta sea depositada por sistema nervioso. Cuantos más sentidos se encuentren implicados en el aprendizaje del lenguaje más información será recibida y más sencillo será su almacenamiento, lo que hará más fácil su aprendizaje. Incorporar el movimiento en el proceso de adquisición del lenguaje, aumentará la probabilidad de que esa información que se transmite sea convenientemente recibida y asimilada. Entendemos, por tanto, que es fundamental aumentar el aprendizaje sensorial implicando el mayor número de sentidos. El aprendizaje Kinestésico contribuye a la retención de la información, a su recuerdo, a su procesamiento y a su transmisión posterior. Se trata de un aprendizaje más significativo.

Creemos firmemente que se debe considerar la introducción del arte en espacios terapéuticos destinados a las alteraciones en el lenguaje ya que la experiencia artística es capaz de modificar los estados de excitación neuronal al generar modificaciones en los impulsos que brotan de los procesos metabólicos, los cuales son los encargados de transportar "la energía que requieren las neuronas para cumplir sus función sináptico-comunicativa" (Luna, 2008, p. 43).

Como hemos comentado anteriormente, la experiencia artística tiene la capacidad de excitar la amígdala, activando nuestra red neuronal y construir y reconstruir mapas neuronales implicados en la construcción del lenguaje. Las neuronas excitadoras se ponen en funcionamiento ante algo novedoso y ante la alteración de los estados de excitación concretos, ya sean visuales o acústicos. Se ha demostrado que las imágenes son influyentes en el bienestar físico y emocional e incluso en el pensamiento. Las manifestaciones artísticas tienen la capacidad de generar imágenes que proceden del inconsciente y al actuar sobre la percepción, activan procesos químicos del cerebro que modifican el estado emocional. A través del arte se liberan emociones que regulan nuestro organismo y nos permite alcanzar un estado de bienestar físico, psíquico y social, como recomienda la Organización Mundial de la Salud en su definición de Salud.

El arte constituye además una vía terapéutica, frente a alteraciones de la comunicación. Estas impiden la expresión de sentimientos, pensamientos y emociones. Mediante el arte, conseguimos expresarnos con total libertad, convirtiéndose así en una vía de descarga ante este tipo de problemas. La experiencia artística, entendida como recurso terapéutico, interviene en el inconsciente y es capaz de activar, como hemos comentado anteriormente, neuronas excitadoras, estimulando la rehabilitación neurológica cuando existen déficits motores o sensoriales. A través de la experiencia significativa que implica la experiencia 
artística, el individuo estimula su proceso perceptivo mediante la devolución de imágenes desde su inconsciente y al mismo tiempo reorganiza pensamientos desarticulados al hacerlos conscientes.

$\mathrm{El}$ arte como terapia busca indagar en el conocimiento interno del ser humano a través de las emociones estéticas y su expresión, especialmente cuando el ejercicio de la palabra no basta o no es suficiente. En arteterapia, el proceso se impone al resultado final:

Observar detenidamente lo que hallamos en ese camino, en el que se pueden encontrar escollos que hay que aprender a solventar; encontrar el momento de descanso para poder contemplar lo que se está creando; llegar hasta algo nuevo que no conocíamos; poder saber seguir hacia delante... no hay que confundir el producto, el resultado final, con el proceso. El arte es un camino desconocido como proceso dramático que se destruye y renueva sin cesar. (López y Martínez, 2006, pp.13-14)

Sofía et al. (2010) realiza la siguiente cuestión, ¿puede hablarse de arte-terapia o se debería afirmar que todo el arte es terapia?.

Es un error creer que existen algunos teatros-laboratorios. Todos los teatros son laboratorios: en todos se experimenta algo que no es real, pero que es en vista de la denominada realidad. (Cruciani, 1995, p. 92).

Sofía et al. (2010) describe como en Chicago en el año 1999, varios psicoanalistas, dirigidos por el psicoanalista austriaco Kohut (1999), durante sesiones de psicoterapia, escribían las fluctuaciones que se daban entre el terapeuta y el paciente. Estas fluctuaciones, según narraciones del propio Kohut (1999), se debían a las imitaciones de las posturas asumidas por el terapeuta y que el paciente asumía. Podemos decir que estas imitaciones se daban como una correspondencia a espejo. Como explicaremos más tarde, se activan las denominadas neuronas espejo.

El Teatro de Sombras tiene el poder de activar las neuronas espejo. Esto se debe a que el espectador, al visualizar su sombra, de manera inconsciente, pone en funcionamiento su propio sistema motor.

Las neuronas en espejo son grupos específicos de neuronas que se activan, tanto cuando el individuo realiza una acción, como cuando el individuo observa esa misma acción realizada por otra persona. En otras palabras, observar una acción provoca de forma inmediata la activación del mismo "programa motor" neuronal en el observador, "programa motor" que estará activo durante la ejecución de la acción: cuando observamos una acción, la rehacemos en nuestro interior. Quizá inhibamos la extensión espacial y temporal de esa acción, pero en nosotros ya ha tenido lugar una activación muscular que ha cambiado nuestro equilibrio interno y que nos ha enviado una información valiosísima acerca de lo que está sucediendo ante nuestros ojos. Sofía et al. (2010).

Ramachandrán (2012), neurólogo hindú, nos cuenta como uno de sus pacientes, que había perdido trágicamente una de sus manos durante la guerra del golfo, ante determinadas acciones era capaz de sentir su mano ausente, a la que denominaban 
mano fantasma. El paciente al observar como Ramachandrán golpeaba o acariciaba la mano de otra persona sentía esas acciones observadas en su propia mano fantasma.

Este artículo trata de mostrar la importancia que pueden tener las neuronas espejos en procesos terapéuticos en individuos con afectaciones motoras y del lenguaje.

Mediante el Teatro de Sombras ponemos en funcionamiento las neuronas espejo, y estas son capaces de, sin necesidad de desplazar el cuerpo, activar el programa motor. El paciente es el protagonista de la escena, y el profesional le invita a vivenciar el problema a tratar en la terapia en ese lugar imaginario donde se representa la escena. El Teatro de Sombras, constituye el espacio donde se da la acción. Falleti et al. (2010) afirma que en la dramatización existen otros espacios además del escenario. El primero de ellos es un espacio sensorial, donde encontramos un gran número de estímulos. Se trata de la superficie de la epidermis, como superficie sensorial, la superficie personal. Un segundo espacio es el espacio peripersonal, es decir, el que rodea a toda persona. Ambos son espacios que nos hacen individuales, nos aíslan y por supuesto, son espacios que nos pertenecen.

Existe además el espacio interpersonal que es aquel que compartimos, el de las relaciones e intercambios. Falleti et al. (2010) nos descubre otro espacio nuevo, siempre presente en la representación dramática, pero no visible por las personas que presencian la acción dramática. Lo describe como un espacio que se mantiene acotado, como el peripersonal, pero se diferencia de este en ser un espacio existente, interno en el ser humano, al que denomina "espacio de acción compartido" (Falleti et al., 2010, pp. 16-17).

Como explica la dramaturga, existe un lugar compartido por espectador y actor en nuestro interior. Ese lugar lo encontramos, por tanto, en nuestro propio interior guardando la misma secuencia, tanto en el tiempo, como en el espacio. En este lugar, coincide además con el programa motor, que es el encargado de que ejecutemos esas mismas acciones. Se crea así, por tanto, un mecanismo reflejo de las acciones que realizan los demás. Se activan las neuronas espejo en el cerebro de la misma forma que cuando realizamos una acción concreta. De esta manera el cerebro del espectador, al observar la acción del actor, la rehace en su interior y activa su sistema motor. El público presente en la dramatización pone a prueba su equilibrio emocional inconscientemente al revivir la acción. Falleti et al. (2010) relata como el actor al mismo tiempo, a través de una danza invisible, se aloja en el interior del espectador:

En teatro (que es el lugar de las miradas por excelencia), el espectador del patio de butacas está realizando esta danza junto con el actor que está en escena y, de forma conjunta, crean un espacio dinámico compartido de acción que es, al mismo tiempo, una danza o peripecia de intenciones. (pp. 20-21)

Las neuronas espejo fueron descubiertas y localizadas en un área del cerebro de un primate que puede considerarse equiparable al área del lenguaje humana. Se considera que antes de lograr la capacidad de comunicarse de forma lingüística, el ser humano lo hacía mediante gestos. En esta capacidad de comunicación gestual las neuronas espejo son tremendamente útiles, ya que descifran la intención comunicativa de la otra persona y controlan el movimiento de las manos (Rizzolatti, 2006). A día de hoy conocemos que al hablar y al escuchar, todas esas áreas del cerebro se activan. Rizzolatti (2006) en la década de los noventa, junto a su equipo de la Uni- 
versidad de Parma, consigue localizar el lugar donde se encuentran las neuronas espejo y su sistema. El estudio lo realiza primero en primates y posteriormente en seres humanos. Tras varios estudios finalmente localizan las neuronas espejo, ubicándolas en la corteza frontal inferior del cerebro, muy cercanas al área del habla. La proximidad y cercanía facilita el estudio sobre la relación existente entre la imitación de sonidos y gestos con el lenguaje. Este equipo de la Universidad de Parma consigue, mediante este estudio, descubrir una zona dónde coincide tanto la precepción como la ejecución de una acción.

Otros estudios científicos han hallado neuronas espejo en las cortezas motora y parietal. Estas neuronas comparten las características básicas pero tienen funciones diversas. Las neuronas localizadas en la corteza parietal, están implicadas en la interpretación de intención de los gestos. Tanto al percibir un gesto en la otra persona, como en el instante en el que uno mismo ejecuta el gesto, las neuronas espejo se activan. Las neuronas espejo son originariamente descubiertas en el área descrita anteriormente en el cerebro del macaco, pero existen evidencias que confirman encontrarse en el cerebro humano y como intervienen en diversas funciones cognitivas, como la empatía o la interpretación de acciones. En lo que nos atañe a este artículo, también son asociadas a la función del lenguaje. Las áreas neurales en las que se han encontrado esas evidencias de su posible existencia dentro del cerebro humano son el lóbulo parietal inferior rostral y el pars opercularis. También en la ínsula, cíngulo anterior y giro temporal superior. Estudios con pacientes, con afectación de la comunicación social, no poseen una activación normal de las neuronas espejo (Enticott et al., 2012).

Estos estudios nos revelan la importancia de las neuronas espejo en la producción del lenguaje, y hace que nos planteamos el Teatro de Sombras como un "espacio compartido" (Falleti, 2010, pp. 16-17) en el que la experiencia intersubjetiva que implica vivenciar la sombra, logra activar el programa motor e implica también la producción del lenguaje.

Schranz (2010) detalla como el lenguaje se basa en el sistema de las neuronas espejo y en la capacidad que promueven las neuronas espejo para la imitación. El lenguaje es connatural al ser humano. Pero esta capacidad ha de activarse y desarrollarse. Si a esa capacidad innata le sumamos la adquisición mediante la práctica y la imitación obtendremos una característica que nos define como seres humanos.

\section{Objetivo del estudio}

El objetivo de este estudio es mostrar la existencia de terapias alternativas, no invasivas, provenientes del arte y en concreto de la dramatización, que mediante la estimulación de las neuronas espejo, pueden mejorar la intención comunicativa de personas con afecciones en el lenguaje.

\section{Método del estudio}

Investigación experimental realizada a partir de un estudio de caso extraído de un estudio de caso múltiple. 
El método aplicado está basado en una recogida de datos cuantitativos y cualitativos que nos aportan información necesaria para dar respuestas a las preguntas de investigación predeterminadas.

Mediante la recogida de datos se ha pretendido aportar conocimientos válidos, demostrables y fiables que muestren la mejora en el menor con dificultades motrices y de lenguaje gracias a la intervención del arte.

A través del estudio pretendemos conocer si existe mejora en el área propioceptiva, lingüística y emocional tras la intervención de Teatro de Sombras y como las neuronas espejos encuentran implicadas en este proceso

La investigación evaluativa nos permite establecer juicios de valor en relación a un programa o una intervención realizada. A través de dicha evaluación, podemos medir de manera objetiva tanto el objeto, la situación o el proceso en relación a diferentes juicios de valor. La investigación evaluativa es una manera de evaluar de forma sistemática mediante la aplicación de un método que tiene que ofrecer garantía y debe ser válido y fiable.

En esta investigación se ha aplicado el método de estudio de caso múltiple. Tomando palabras de Yin (2005) el estudio de caso es una "estrategia de investigación que comprende todos los métodos con la lógica de la incorporación en el diseño de aproximaciones específicas para la recolección de datos y el análisis de éstos" (p.133).

El estudio de caso múltiple según Yin (2003), debe comprender la recopilación de datos que muestran los resultados de un mismo estudio aplicado sobre dos o más casos que aportan validez a la investigación y distintas pruebas de su eficacia. Se ha considerado oportuno aplicar este método ya que nos permite estudiar a los nueve casos de manera conjunta e investigar el Teatro de Sombras como un fenómeno concreto (Stake, 1998). Se trata de un estudio evaluativo ya que la recogida de los datos pretende evaluar la intervención de Teatro de Sombras para emitir juicios y conclusiones de la intervención realizada (Cea D'Ancona, 1988).

\section{Muestra seleccionada}

El niño sobre el que se aplica esta intervención, según informe del Servicio de Neuropediatría de un Hospital Universitario de la Comunidad de Madrid, fechado en el 2016, presenta retraso madurativo. Este retraso madurativo provoca una discapacidad del 20\%, según Dictamen Técnico Facultativo del equipo de Valoración y Orientación de la Comunidad de Madrid. El dictamen habla de la necesidad de aplicar Atención Temprana en logopedia, psicomotricidad y psicoterapia. La edad cronológica del Niño es de 4 años y 10 meses. Está cursando $3^{\circ}$ de Educación Infantil en Centro Público de la Comunidad de Madrid. Se encuentra dentro de la modalidad ACNEES en la escuela.

El Niño tiene gran timidez. Al lograr mayor confianza muestra un carácter competitivo. Presenta baja autoestima, es impulsivo, tiene escasa tolerancia a la frustración y presenta dificultad en la fluidez del habla. Presenta dificultad para mantener la atención o un tema de conversación prolongado, saltando de un tema a otro. Sus respuestas se pueden considerar precipitadas. Presenta dificultad en la articulación de palabras y tiende a acelerarse al hablar. Durante las conversaciones aparecen de forma asidua repeticiones de sílabas e incluso de palabras 
completas que afectan a la fluidez del lenguaje. Además tiene dificultas para permanecer callado. Presenta movimientos bizarros durante las conversaciones, como meterse las manos constantemente en la boca o cerrar los ojos con fuerza. Presenta una gran inquietud motora. Destaca que tiene problemas al sentir su cuerpo o disociar una parte de otra. Es muy literal en la interpretación de los mensajes y posee dificultades para realizar inferencias. Tiene un discurso desorganizado y, en ocasiones, incoherente. Presenta un autoconcepto deficiente. Tiene unos intereses que en ocasiones resultan restringidos y repetitivos. Le cuesta la relación con sus iguales.

\section{Instrumentos utilizados}

Los instrumentos de evaluación y medición utilizados en esta intervención son cuatro.

- Se realiza una doble entrevista, antes y después de realizar la intervención en las que se efectúa una observación directa y toma de datos.

- Durante la entrevista se realiza la medición de los decibelios, mediante un sonómetro.

- También se toma medida de la frecuencia cardiaca utilizando un pulsioxímetro.

- Finalmente se utiliza un cuestionario destinado a la familia.

Pasamos, a continuación, a describir cada uno de ellos:

Se realizan dos entrevistas a las que fue sometido el niño, con la finalidad de registrar el máximo número de datos que muestren el estado emocional del menor, antes y después de la intervención de Teatro de Sombras. La primera de las entrevistas, o entrevista Pre, es previa a la intervención y se realiza el 16 de Febrero del 2017. La Segunda, o entrevista Post, se realiza ya cumplidas las treinta sesiones establecidas y es realizada el 10 de Mayo del 2017. Las entrevistas tuvieron una duración de tres minutos y fueron realizada por tres miembros que participan exclusivamente en las entervistas y son ajenos a su entorno habitual, con la finalidad de que el menor no obtuviera familiaridad con ellos y de esta forma no distorsinar las entrevistas. Durante las msimas se realizaron al niño preguntas sencillas de la vida cotidiana, que no fueran complejas para él, como son preguntas personales, del entorno familiar, o del entorno escolar.

- Como primera herramienta utilizada a lo largo de las entrevistas se aplicó la observación directa, estrategia que nos facilitó la recogida de datos y de información, valorando así el comportamiento del niño.

Prestamos especial atención a la Kinesia a la que podemos definir como el lenguaje no verbal o lenguaje corporal expresado a través de la postura corporal, los gestos, expresiones faciales... Mediante la observación directa en las entrevistas se llevaron a cabo los registros de los siguientes estados corporales, cuya finalidad es reconocer la intención comunicativa y el estado emocional del niño:

Contacto ocular. El número de contactos oculares mantenidos durante las entrevistas nos aportan una información cuantitativa relevante en este estudio, ya que el contacto ocular es una herramienta no verbal altamente significativa, transmitiéndonos el grado de seguridad del sujeto. 
Manos: Escondidas (muestra inhibición, represión o miedo), manos o dedos entrelazados (pone de manifiesto estados de nerviosismo o ansiedad) o manos mostradas (trasmite seguridad).

Postura corporal: Relajado-estático (muestra seguridad), tenso-estático (muestra ansiedad-miedo), estático-nervioso (muestra estados elevados de ansiedad-nerviosismo) y movimiento continuo (muestra nerviosismo propio de estados de ansiedad).

Movimientos bizarros: pueden aparecer como características propias del niño o ser provocados por elevados estados de ansiedad, inseguridad, miedo o nerviosismo.

- Al mismo tiempo, durante el transcurso de las entrevistas utilizamos la segunda herramienta. Se trata de un Sonómetro Optimus Green que nos posibilita el registro de la intensidad de volumen medida en decibelios (db).

Mediante estas dos herramientas, se registraron los siguientes datos Pre y Post del niño:

Lenguaje verbal. Mediante la observación directa como estrategia de recogida de información se toman los siguientes registros:

Tono al hablar: ascendente-lineal-descendente.

Ritmo al hablar: Taquilálico (fluidez del habla excesivamente acelerado), bradilálico (velocidad excesivamente lenta en la fluidez del habla), o normal.

Como ya hemos comentado, para registrar el volumen del niño se aplicó un sonómetro, instrumento de medida de decibelios (db). Hemos considerado, a efectos de este estudio, como volumen bajo cuando se mide hasta un máximo de cuarenta y cinco db, de cuarenta y cinco a cincuenta y cinco db lo hemos considerado como volumen medio y a partir de cincuenta y cinco db se consideró un volumen alto.

El volumen de la conversación nos indicará una mayor o menor seguridad en sí mismo al comunicarse con el interlocutor o el grado de ansiedad que le supone al menor expresarse con su dificultad.

- Como tercera herramienta utilizada usamos un medidor de la frecuencia cardiaca (FC) Pulse Oximeter. Registro de FC:

Consideramos que para profundizar en la investigación es necesario el registro de datos fisiológicos. La toma de mediciones del estado de la frecuencia cardíaca (FC) del niño durante el discurso en púbico nos ofrece datos significativos del aumento o disminución de la ansiedad.

Antes de comenzar la entrevista para la toma de mediciones, debemos asegurarnos de que no existen factores que alteren el normal comportamiento del niño. Estos factores podrían ser la toma de algún tipo de medicamentos o factores fisiológicos determinantes, como falta de sueño. Es importante que el niño no esté familiarizado con las personas que realizan la entrevista a las que solo verá en las entrevistas y en un intervalo muy corto de tiempo.

Reflejar en gráficas el aumento de la FC del niño durante la entrevista y concretamente durante sus conversaciones, significa obtener datos objetivos del aumento o disminución del miedo y la ansiedad. Para la toma de medida fácil y no invasiva de la FC del niño se utiliza un Pulse Oximeter MCS-50F de muñeca. Este modelo es utilizado para estudios de hipoxia y apnea de sueño. Resulta un instrumento de medida idóneo para el registro de la FC ya 
que el niño puede llevarlo cómodamente en la muñeca a lo largo de toda la entrevista.

- La cuarta herramienta utilizada para la recogida de información en esta investigación fue el cuestionario de respuestas abiertas destinado a la familia del niño. La información recopilada mediante este cuestionario familiar nos aporta información relevante ya que la familia pudo mantener una observación directa del estado físico y emocional del menor, antes y después de la intervención de Teatro de Sombras.

Este cuestionario está compuesto por cuatro preguntas abiertas destinado a los familiares del niño y un espacio en blanco, reservado para sus observaciones y opiniones.

En una primera cuestión se les pregunta por el estado de ánimo con el que acude el niño a las terapias según avanzan las sesiones y el estado de ánimo al salir de las mismas. Por otro lado se les cuestiona por las dificultades más significativas que presenta el niño de forma habitual. Como tercera cuestión se les pide, según su perspectiva, y tratando de ser lo más objetivos posibles, que enumeren si han observado mejoras y si estas han ido perdurando en el tiempo. Por último, se ofrece un espacio en blanco reservado para que los familiares emitan libremente opiniones $\mathrm{u}$ observaciones sobre las intervenciones realizadas y para que valoren como creen que las intervenciones han podido influir sobre las dificultades observadas en sus hijos.

\section{Procedimiento}

Mediante el análisis de los datos registrados en las entrevistas pretendemos conocer si existe mejora en la intención comunicativa y el estado emocional del niño tras la intervención de Teatro de Sombras. La intervención se realiza en un centro privado de Atención Terapéutica de la Comunidad de Madrid en el que el menor está recibiendo tratamiento para mejorar las distintas dificultades que se han comentado con anterioridad. El niño fue sometido a una primera entrevista el 16/02/2017 en dicho centro. Las entrevistas nos proporcionan información directa para registrar datos implicados en el lenguaje verbal (tono, ritmo, volumen) en la Kinesia (número de contactos oculares, manos, postura corporal y movimientos bizarros) que no pueden ser obtenidos de otra forma. Además obtenemos datos sobre la frecuencia cardiaca que nos informarán sobre estado de estrés y/o ansiedad durante las entrevistas. Estas entrevistas tuvieron una duración de tres minutos y fueron realizadas por tres profesionales (una investigadora del ámbito del Arteterapia que cumplió la función de entrevistador y dos terapeutas ajenos al entorno del niño que ejercieron como observadores sistemáticos y fueron entrenados previamente). Las entrevistas se componen de preguntas personales conocidas por el niño y sobre situaciones cotidianas. Fueron preguntas sencillas, como edad, lugar de residencia, preguntas relacionadas con el entorno familiar o escolar o preguntas sobre intereses personales.

Los datos obtenidos en esta primera entrevista, aperecen reflejados en la tabla 1. 
Tabla 1. Recogida de datos Pre. 16 de Febrero del 2017, Medida del lenguaje verbal, FC, y Kinesia.

\begin{tabular}{|l|l|l|l|l|l|}
\hline \multicolumn{2}{|c|}{ Lenguaje Verbal } & \multicolumn{2}{|c|}{ Frec. cardiaca } & \multicolumn{2}{c|}{ Kinesia } \\
\hline Tono & Ascendente & \multirow{2}{*}{$\begin{array}{l}N^{\mathbf{o}} \text { contactos oculares/ } \\
\text { Mirada }\end{array}$} & 11 \\
\cline { 5 - 6 } Ritmo & Taquilálico & $\begin{array}{l}\text { Mulsaciones } \\
\text { FC) }\end{array}$ & 110 & Manos & Escondidas \\
\cline { 5 - 6 } Volumen & $\begin{array}{l}56(\mathrm{db}) \\
\text { Medio/Alto }\end{array}$ & & Postura & Movim. continuo \\
\hline & & & Movim. Bizarros & Cierra fuerte los ojos \\
\hline
\end{tabular}

Finalizado el registro de datos PRE, se llevan a cabo las treinta sesiones de intervención de Teatro de Sombras. Estas se realizan entre el 19 de Febrero y el 9 de Mayo. La duración de cada sesión fue de cincuenta minutos y se aplicaron tres días a la semana. Durante las treinta sesiones, con el fin de lograr el objetivo propuesto en esta investigación, se desarrollaron sesenta y tres juegos de sombras con diferentes contenidos:

- Estimulación del ritmo.

- El uso del cuerpo como instrumento para la expresión de sentimientos.

- Otras formas o recursos del cuerpo para expresarse como el gesto y el movimiento.

- La relación entre el espacio y el movimiento.

- El lenguaje corporal y su relación con otros lenguajes.

Con el fin de afianzar estos contenidos se busca conseguir los siguientes objetivos procedimentales y conceptuales a lo largo de la intervención:

- Investigar, experimentar y explorar con la sombra corporal para descubrir los recursos expresivos que nos proporciona.

- Identificar estados de ánimo y sensaciones a través de las sombras.

- Explorar la sombra a través del movimiento para integrar las propiedades del movimiento en la práctica motriz.

- Adecuar el cuerpo y el movimiento a la reproducción de ritmos.

- Trabajar las expresiones de sentimientos a través de la sombra y el movimiento para abrir nuevas vías de comunicación y representación.

- Comunicar a través del movimiento.

Estos sesenta y tres juegos de sombras están también planteados para una estimulación multisensorial abarcando diferentes áreas y recrear así movimientos inspirados en los patrones básicos de los primeros desplazamientos: el patrón homolateral, el arrastre, el gateo, el patrón cruzado y ejercicios de braquiación y de marcha, movimientos que unidos estimulan el sistema vestibular, el control espacial y la propiocepción (Doman, 1960).

Antes de comenzar las sesiones se dedicó un tiempo al calentamiento y al finalizar se procedió a la relajación. Este tiempo empleado ayuda al niño a aprender a relajarse y tomar conciencia corporal. 
En los juegos propuestos, el niño detecta los cambios posturales que se van produciendo y así interpreta y comprende los mensajes corporales que visualiza a través de la sombra.

En algunos de los juegos propuestos, el niño sincronizó el movimiento de su cuerpo a ritmos que iban apareciendo en cada momento. De esta forma, el niño pudo ir definiendo su esquema corporal. Pudo además definir su preferencia lateral a medida que progresaba su agilidad, velocidad y equilibrio. Pudo ir distinguiendo izquierda-derecha e ir trabajando la noción del tiempo dependiendo de la velocidad.

Durante las sesiones de Teatro de Sombras se utilizaron los siguientes recursos materiales:

Una pantalla que hacía de soporte para la proyección de las sombras

Un foco de luz o proyector.

Como material adicional se utilizó la música como elemento de acompañamiento en las sesiones. Le facilitó al niño la recreación de movimientos estimulando diferentes áreas. El acompañamiento de la música hace posible que aparezcan diferentes estados emocionales.

Finalmente, para poder verificar si existe mejora en la conciencia corporal, la intención comunicativa y el estado emocional del niño tras la intervención de Teatro de Sombras, se realiza la segunda entrevista, o entrevista Post. Esta se realiza el 10 de Mayo del 2017. Como se comentó anteriormente, en esta entrevista intervienen nuevos profesionales (una investigadora del ámbito del Arteterapia que cumplió la función de entrevistadora y dos nuevos terapeutas, ajenos al entorno del niño, que ejercieron como observadores sistemáticos y fueron entrenados previamente). La entrevista Post estuvo compuesta por nuevas preguntas en relación a aspectos similares a la primera entrevista (aspectos personales, relacionadas con el entorno familiar y escolar y preguntas de intereses personales).

Durante la entrevista Post se registran los siguientes datos representados en la tabla 2 :

Tabla 2. Recogida de datos Post. 9 de Mayo del 2017., Medida del lenguaje verbal, FC, y Kinesia.

\begin{tabular}{|l|l|l|l|l|l|}
\hline \multicolumn{2}{|c|}{ Lenguaje Verbal } & \multicolumn{2}{c|}{ Frec. cardiaca } & \multicolumn{2}{c|}{ Kinesia } \\
\hline Tono & Ascendente & \multirow{2}{*}{$N^{0}$ Pulsaciones } & 76 & $\begin{array}{l}\mathbf{N}^{\mathbf{0}} \text { contactos oculares/ } \\
\text { Mirada }\end{array}$ & 13 \\
\cline { 1 - 3 } Ritmo & Taquilálico & (FC) & & Manos & Mostradas \\
\cline { 1 - 3 } Volumen & $50(\mathrm{db}) /$ Medio & & & Postura & Movim. continuo \\
\hline & & & & Movim. Bizarros & Cierra fuerte los ojos \\
\hline
\end{tabular}

\section{Resultados obtenidos}

El Niño ha mostrado elevados niveles de ansiedad en la primera entrevista. Se registra una FC máxima de 110 pulsaciones. Durante la entrevista el Niño se muestra muy inquieto, con constantes movimientos y escondiendo las manos. En algunas ocasiones nos interrumpe respondiendo antes de que finalicemos las preguntas. Durante 
la entrevista el lenguaje se torna ininteligible y nos cuesta comprender su lenguaje verbal, ya que su ritmo es taquilálico y desorganizado. Cuando no encuentra las palabras adecuadas o se bloquea en su discurso, cierra con fuerza los ojos. El tono durante la entrevista es ascendente y se registran un volumen máximo de $56 \mathrm{db}$, por lo que consideramos el volumen como medio/alto. Se registran 11 contactos oculares.

En la segunda entrevista el Niño se muestra menos inquieto, se registra una FC máxima de 76 pulsaciones, lo que nos indica que el niño se encuentra más tranquilo y seguro, a pesar de que las personas que intervienen en la entrevista no han participado en las sesiones ni han coincidido en la entrevistas. El ritmo de su discurso sigue siendo taquilálico y el tono ascendente pero se registran $50 \mathrm{db}$ de máxima, descendiendo por tanto su volumen en relación a la primera entrevista, lo que indica menor ansiedad a la hora de transmitir y mantener una conversación. Aumentan sus contactos oculares, registrándose hasta 13, lo que denota confianza. Durante la segunda entrevista se observa que cierra en ocasiones los ojos con fuerza, aunque en menor medida.

\section{Valoraciones externas}

Pasamos a describir a continuación las valoraciones externas tras la intervención. Estas son el cuestionario facilitado a los familiares y la valoración del centro externo dónde se estaban tratando las dificultades del menor.

Como ya comentamos con anterioridad, se facilita un cuestionario a las familias con diferentes cuestiones. En primer lugar se les pide que describan el estado del Niño en el momento de acudir a las sesiones de Teatro de Sombras. A estas contestan que el Niño "acude muy contento y eufórico”. Como segunda cuestión, solicitamos que describan el estado de ánimo del Niño al salir de las sesiones, contestando que "feliz, sale muy contento y le encanta pasar tiempo con sus compañeros de teatro", refieren que "notan que se relaciona mejor con sus compañeros de teatro que con los niños del colegio". Les pedimos que describan brevemente las dificultades más notorias del Niño, respondiendo que "le cuesta expresarse, que a veces se lía y tartamudea. Le cuesta todo lo relacionado con psicomotricidad, subir y bajar escaleras, saltar...". Les pedimos que, respecto a las dificultades señaladas, señalen si han observado algún cambio tras las sesiones de Teatro de Sombras, comentando que " $a$ la hora de expresarse si notamos que habla mejor y también se relaciona mejor con los niños de teatro". Finalmente les pedimos que señalen si los cambios han perdurado en el tiempo y nos contestan "que de momento si". En observaciones comentan que se "van muy contentos con todo, describen a la profesional como muy cercana y atenta y comentan que los niños están encantados". Análisis de kas fanmiliar or laura

Por otra parte, se pide al centro de atención terapéutica de la Comunidad de Madrid dónde el menor está recibiendo tratamiento, la valoración de la intervención realizada.

El centro, en palabras de su directora, valora como mediante el teatro de sombras se ha ofrecido a tener una experiencia significativa a un menor que mostraba serios problemas en construir, pensar, imaginar, jugar y un bajo tono muscular que se manifestaba en una inhibición de su libre movimiento. Asegura haber observado una mejoría en el ámbito psicomotor que aúna el cuerpo con el funcionamiento subjetivo. 
Según n sus palabras, la propuesta ha supuesto una puesta en escena del cuerpo para armar una representación en movimiento a través de historias y juegos que se han llevado a cabo a través de la proyección de sombras. Destaca el interés de la intervención, ya que en la constitución inicial del sujeto es fundamental la imagen en movimiento del cuerpo reflejada en el espejo y diferenciada de la imagen del cuerpo del otro. Finaliza su valoración destacando que el trabajo realizado durante las 30 sesiones ha ayudado al niño a estructurarse de manera subjetiva y que se ha mostrado más espontáneo, con un aumento del juego simbólico y con mayor conciencia de la imagen corporal, que le ha facilitado la posibilidad de vivenciar y experimentar y, por tanto, de aprender, ya que el aprendizaje no es posible sin movimiento y emoción, y claramente es dificultado por la inhibición.

\section{Conclusión}

A partir de los resultados obtenidos, y de las valoraciones externas, tanto de la familia como del centro de atención terapéutica, estamos en la convicción de que puede ser efectivas terapias alternativas en el tratamiento de afecciones del lenguaje. Estas intervenciones podrían ser capaces de mejorar en la intención comunicativa y en el estado emocional y anímico de las personas intervenidas.

La dramatización ha ofrecido al niño un nuevo canal de comunicación donde incluso el propio silencio se ha transformado en escucha de la dilatación de su propia conciencia. Hemos podido observar como durante las sesiones de dramatización el niño permanecía inmerso en el juego dramático mientras la tensión muscular e inquietud motriz se disolvían en un espacio de libertad creativa. El niño en el Teatro de Sombras ha mostrado una predisposición a exponerse frente a su propia sombra y frente al grupo. En la sombra, ha descubierto un espacio entre la realidad y no realidad donde ha podido expresar sus emociones y liberar su voz oprimida. La exploración con su propia sombra ha significado una exploración interior que le ha permitido exteriorizar conflictos reprimidos. En nuestro acompañamiento de escucha hemos podido observar como la tensión emotiva se ha transformado en emoción creativa. Hemos contemplado como el miedo a hablar y el miedo a ser observado, ha ido desapareciendo en el trascurso de las sesiones. Ahora el niño, ha encontrado en su propia sombra un lugar donde mirarse para ser visto y ser reconocido. El Teatro de Sombras le ha ofrecido un lugar de experimentación con su propio cuerpo, sintiéndolo y vivenciándolo ha podido conectar con él para autorregularse.

El objetivo principal que se planteó en esta investigación fue mostrar la importancia que pueden tener las neuronas espejos en procesos terapéuticos en individuos con afectaciones motoras y del lenguaje y la posibilidad de desarrollar sesiones de Teatro de Sombras desde una visión artística y terapéutica. De esta forma, se otorga al niño que sufre trastornos del lenguaje, una oportunidad de liberar su cuerpo y verbalizar sus emociones. Dicho objetivo fue propuesto con la intención de esclarecer la hipótesis general de investigación que plantea como el diseño de una terapia que incluya el cuerpo con sus variantes semánticas a través de prácticas artísticas mejora la comunicación en niños afectados por trastornos del lenguaje.

Mediante el Teatro de Sombras, como un espacio terapéutico, y a través de la expresión artística y corporal que el niño ha experimentado, ha vivenciado de manera integral su cuerpo, experimentando con su sombra corporal. Proyectando 
el cuerpo en la sombra, el cuerpo interviene como intermediario entre la emoción y la conciencia, entre el cuerpo y la mente, como una unidad indivisible. El niño, mientras dramatiza su realidad, va adquiriendo conciencia de su cuerpo mientras indirectamente reorganiza su sistema sensorio motriz. Revivir etapas del desarrollo que fueron bloqueadas a través de la dramatización, significa favorecer indirectamente el lenguaje.

Así pues creemos firmemente que favoreciendo la conciencia corporal mediante los juegos de sombras se abre una puerta a alternativas para mejorar el estado psicomotor, emocional y social de pacientes afectados por dificultades semejantes a las del niño objeto de este estudio.

Concluimos, por tanto, que el Teatro de Sombras y la estimulación de las neuronas espejo, como propuesta terapéutica aplicada en el menor afectado por trastornos del lenguaje, nos muestra la posibilidad de una intervención diferente, no invasiva, enfocada al juego, a la creación y a la emoción, que podría ser eficaz ante problemas o dificultades tanto físicas, como psicológicas o emocionales. Por lo tanto, consideramos que sería conveniente profundizar en su estudio y considerarse en un futuro la posibilidad de la inclusión del Teatro de Sombras en los programas de Necesidades Educativas Especiales como un recurso terapéutico.

\section{Referencias bibliográficas}

SBrook, P. (2002). La puerta abierta: Reflexiones sobre la interpretación y el teatro. Alba editorial.

Cea D'Ancona, M. (1998). La organización de la investigación en Metodología cuantitativa. Estrategias y técnicas de investigación social. Madrid: Editorial Síntesis, pp. 81-122.

Chavarría, M. (2015). La eficacia de la creatividad: Creactívate. Madrid: ESIC.

Cruciani, F. (1995). Sulla scienza di Stanislavskij, en Registi pedagoghi e comunità teatrali nel Novecento. Roma: Editori \& Associati.

Cruz, V., Caballero, P., y Ruiz, G. (2013). La dramatización como recurso didáctico para el desarrollo emocional. Un estudio en la etapa de Educación Primaria. Revista de Investigación Educativa, 31(2), 393-410.

Damasio, A. (2011). El error de Descartes: La emoción, la razón y el cerebro humano. Barcelona: Destino.

Doman. J., Spitz, E. B., Zucman, E., Delacato, C. H. \& Doman, G. (1960). Children with severe brain injuries. Journal of the American Medical Association, 174, 257-262.

Emunah, R. (1994). Acting for Real: Drama Therapy, Process, Technique and Performance. Levittown, PA: Brunner Mazel.

Enticott, P., Kennedy, H., Rinehart, N., Tonge, B., Bradshaw, J., Taffe, J., Daskalakis, Z., y Fitgerald, P. (2012). Mirror neuron activity associated with social impairments but not age in autism spectrum disorder. Biological Psychiatry, 71, 427-433.

Falleti, C., Mirabella, G., Pardiso., Pradier, J.M., Schranz, J., Czertok, H., Mariti y Sofía, G (coordinador). (2010). Diálogos entre teatro y neurociencias. Bilbao: Artezblai.

Kohut, H. (1999). La restauración del si-mismo. México: Editorial Paidós.

López, M. y Martínez, N. (2006). ARTETERAPIA, Conocimiento interior a través de la expresión artística. Madrid: Editorial Tutor.

Luna, J. (2008). Complejidad en Educación. Valencia: Nau Llibres 
Martín, I., y López Pastor, V. M. (2007). Teatro de sombras en educación infantil: Un proyecto para el festival de navidad. Retos: Nuevas Tendencias En Educación Física, Deporte y Recreación.

Moreno, J. (1995). Las bases de la psicoterapia. Buenos Aires: Hormé.

Ramachandran, V \& Oberman, L. (2006). Broken mirrors: a theory of autism. Sci Am 2006; 295: 39-45. 4.

Rizzolatti, G. (2006). Las neuronas en espejo: los mecanismos de la empatía emocional. Barcelona: Paidós.

Schranz, J. (2010). Hablemos de ampollas. En Falleti, C., Mirabella, G., Pardiso., Pradier, J.M., Schranz, J., Czertok, H., Mariti y Sofía, G (coordinador). (2010). Diálogos entre teatro y neurociencias. Bilbao: Artezblai.

Sofía, G. (2010). Sobre intenciones y resonancias. La relación actor-espectador entre teatro y neurociencias. En Falleti, C., Mirabella, G., Pardiso., Pradier, J.M., Schranz, J., Czertok, H., Mariti y Sofía, G (coordinador). (2010). Diálogos entre teatro y neurociencias. Bilbao: Artezblai.

Stake, R. (1998). Investigación con estudio de casos. Madrid: Morata.

Yin, R. (2003). Applications of case study research. London: Sage. 